to improve sepsis survivors' outcomes. Highlighting profiles of sepsis survivors can inform prioritization and tailoring of HHC interventions for this vulnerable group.

\section{CHANGE IN VULNERABILITY AMONG OLDER CARDIAC ADULTS AFTER HOSPITAL DISCHARGE: ROLE OF POST-ACUTE HOME HEALTH}

J. Wang ${ }^{1}$, M. Dietrich ${ }^{2}$, S. Bell ${ }^{3}$, C. Maxwell ${ }^{4}$, S. Simmons ${ }^{5}$, S. Kripalani ${ }^{3}$, 1. University of Rochester, 2. Vanderbilt University School of Nursing, 3. Department of Medicine, Vanderbilt University Medical Center, 4. Vanderbilt University, School of Nursing, 5. Center for Quality Aging, Vanderbilt University Medical Center

Vulnerability to functional decline after hospitalization is high among older cardiac patients. Few studies have quantified the changes in vulnerability after hospital discharge among patients who do or do not receive post-acute services (i.e., home health care). In this prospective study, we aimed to: 1) compare changes in vulnerability after hospital discharge in older patients discharged home with/without a home health care (HHC) referral, and 2) examine factors associated with changes in vulnerability during this period. The sample included 834 patients aged 65 or older, hospitalized for acute coronary syndromes and/or acute decompensated heart failure. Vulnerability was assessed using the Vulnerable Elders Survey-13 at baseline (prior to hospitalization), 30 days and 90 days post-discharge. Linear regression analyses with propensity score matching showed that relative to non-HHC-referred patients, patients referred to postacute $\mathrm{HHC}$, despite having more vulnerability at baseline and delayed recovery in vulnerability in the initial 30 days after hospital discharge, showed comparable improvement in their vulnerability at 90 days after hospital discharge. Baseline vulnerability and HHC referral accounted for $14 \%$ $16 \%$ of the variance in 90 -day vulnerability change after hospital discharge $(\mathrm{p}<0.001)$. Patient characteristics (age, number of outpatient visits in the past 12 months, depressive symptoms, race/ethnicity [African American]) contributed $6 \%$ variance $(\mathrm{p}<0.001)$. Our findings suggest that post-acute $\mathrm{HHC}$ facilitates functional recovery in the most vulnerable older patients, however additional studies are warranted. Future research should examine the impact of the timing and intensity (frequency, visit pattern and types of services) of post-acute HHC on vulnerability changes after hospital discharge.

\section{CLINICAL PROFILES OF SEPSIS SURVIVORS IN HOME HEALTH CARE WITH COGNITIVE SYMPTOMS AND DEMENTIA}

M. Ryvicker ${ }^{1}$, L. Jordan ${ }^{1}$, K. Bowles ${ }^{2}$, P. Feldman ${ }^{1}$, C. Murtaugh ${ }^{1}$, 1. Visiting Nurse Service of New York, 2. University of Pennsylvania School of Nursing

Sepsis is a critical public health problem in the U.S., with heightened risk of sepsis severity shown to affect the growing population of older adults with Alzheimer's disease and related dementia (ADRD). As part of a larger study on survivors of sepsis receiving home health care (HHC), we compared clinical and demographic profiles and health outcomes of sepsis survivors across three subgroups: patients with ADRD; patients without a known ADRD diagnosis but with cognitive symptoms identified on the HHC clinical assessment; and patients with neither of these characteristics ("reference"). Using Medicare claims and HHC assessment data on a national sample of 165,228 sepsis survivors discharged from the hospital to HHC within one year, we examined associations between ADRD/cognitive status and 6-month outcomes. Thirty-one percent had ADRD, while $11 \%$ had cognitive symptoms without an ADRD diagnosis. The ADRD group was older $(42 \%$ age $85+$ compared to $19 \%$ with cognitive symptoms and $16 \%$ of the reference), had a greater proportion female $(60 \%$ vs. $54 \%$ and $53 \%$, respectively), and had a greater proportion with urinary tract/ kidney infection as the source of sepsis $(52 \%$ vs. $38 \%$ and $34 \%$, respectively). Compared to the reference, the ADRD group had greater odds of re-hospitalization within 6 months in multivariable regression $(\mathrm{OR}=1.32 ; 95 \% \mathrm{CI}=[1.29,1.36])$. Both the ADRD group and cognitive symptoms group had greater odds of nursing home admission. The subgroups of sepsis survivors with ADRD and cognitive symptoms have unique profiles and risk factors that may be used to inform $\mathrm{HHC}$ interventions for these vulnerable patients.

\section{CHARACTERISTICS OF LGBT CAREGIVERS OF OLDER ADULTS: RESULTS FROM THE NATIONAL CAREGIVING IN THE U.S. 2015 SURVEY}

J. Anderson ${ }^{1}$, J. Flatt ${ }^{2}, 1$. University of Tennessee, 2.

University of California-San Francisco

Using data from the Caregiving in the U.S. 2015 national survey, we compared characteristics of LGBT and non-LGBT caregivers of older adults and explored predictors of caregiver strain. LGBT caregivers were younger, more racially/ ethnically diverse, and less frequently a spouse/partner compared with their heterosexual counterparts. LGBT caregivers more frequently reported helping with medical nursing tasks and reported higher levels of financial strain. While LGBT identity was not an independent predictor of greater strain, characteristics more frequently reported by LGBT caregivers were, for instance helping with medical nursing tasks. Additionally, LGBT individuals who were children of the older adult were more likely to report higher levels of emotional strain than other relationship types. This is one of the first national studies to compare the characteristics and experiences of LGBT caregivers of older adults to their non-LGBT caregiver counterparts. Future research is needed to understand better the physical, emotional, and financial challenges faced by LGBT caregivers, as well as their use of caregiver resources. In addition, future policies aimed at improving caregiver support and services for LGBT caregivers and nontraditional family members should be considered.

\section{SESSION 2430 (SYMPOSIUM)}

IMPLEMENTING FUNCTION FOCUSED CARE IN AL: MEASUREMENT CHALLENGES AND SOLUTIONS

Chair: B. Resnick, University of Maryland School of

Nursing, Baltimore, Maryland

Discussant: A. Bonner, Massachusetts State Department of

Health, Boston, Massachusetts

Residents in Assisted Living (AL) engage in limited amounts of physical activity and decline functionally more rapidly than their peers in nursing homes. Reasons for decline include resident factors (e.g., comorbidities, age) and 